\title{
INTEGRAL EQUATION FORMULATION OF SOME THREE PART BOUNDARY VALUE PROBLEMS
}

\author{
by W. E. WILLIAMS \\ (Received 18th May 1963)
}

\section{Introduction}

One of the simplest three part boundary value problems is the electrostatic problem for the circular annulus and, at present, there seems to be no method available for obtaining the solution in a closed form. It has recently been shown by the author (1) and Cooke (2) that this problem can be reduced to the solution of a Fredholm integral equation of the second kind. The equation obtained in $(\mathbf{1}, \mathbf{2})$ is fairly simple and is suitable for obtaining a numerical solution but, unfortunately, it cannot be solved iteratively to give a simple form of solution valid for small values of the ratio (inner radius/outer radius).

An alternative approach to the annulus problem has been developed by Gubenko and Mossakovskii (3). These authors treat the axi-symmetric problem and show that it can be reduced to the solution of a pair of simultaneous Fredholm integral equations. These equations have the advantage that they can be solved iteratively to yield an expansion for the solution in powers of (inner radius/outer radius). The method adopted in (3) is to split the original problem into two separate ones and then use suitable integral representations to reduce these problems to ones in two dimensional potential theory. These latter problems are then reduced in an ingenious fashion to the solution of a pair of coupled Fredholm integral equations.

Collins (4) in considering the axi-symmetric electrostatic problem for an annular cap has used the approach of (3) to reduce this problem to the solution of a pair of integral equations. Collins formulates the problem as the solution of a set of triple series equations involving Legendre functions. The author in a recent paper (5) discussing the relationship between certain dual and triple series equations and dual and triple integral equations has shown that the equations derived by Collins may be obtained by a direct change of variable from those of Gubenko and Mossakovskii. Collins also considers a more general set of triple series equations which he shows can be reduced to the solution of four simultaneous integral equations.

It is possible by using an analysis similar to that of Collins to adapt the technique of (3) to solve three part boundary value problems of the type which can be reduced to triple integral equations involving Bessel Functions. There are, however, certain boundary value problems for the annulus which cannot be 
easily formulated in this way. One example of such a problem is the electrostatic one for the annulus in a cylinder.

In solving the corresponding type of problem for a circular disc an integral equation formulation has proved fruitful (6) and it therefore seems worthwhile to consider an integral equation formulation of some three part boundary value problems and this is the object of the present paper.

In Section 2 the electrostatic problem for the annulus is formulated as a Fredholm integral equation of the first kind and the approach of Gubenko and Mossakovskii is then adapted to reduce this integral equation to the solution of a pair of coupled Fredholm equations of the second kind. For the case of axial symmetry the equations obtained may be identified with those of (3).

In Section 3 the results of Section 2, together with the approach used in (6), are employed to reduce more general boundary value problems to the solution of four simultaneous integral equations. The problems treated in Section 3 include those which can be reduced to triple integral equations and also others, such as the problem of the annulus in a cylinder, which cannot be formulated in this fashion.

\section{The electrostatic problem for the circular annulus}

The general non-axially symmetric electrostatic problem for a circular annulus of inner radius $b$ and outer radius $a$ reduces to solving an integral equation of the form

where

$$
\int_{b}^{a} f(t) K(\rho, t) d t=\phi(\rho), \quad b<\rho<a,
$$

$$
K(\rho, t)=\int_{0}^{2 \pi} \frac{\cos \mathrm{n} \phi d \phi}{\left(\rho^{2}+t^{2}-2 \rho t \cos \phi\right)^{\frac{1}{2}}}, n \text { an integer. }
$$

Following Gubenko and Mossakovskii (3) we shall assume $\phi(\rho)$ has the expansion

and we shall write

$$
\phi(\rho)=\sum_{n=-\infty}^{\infty} a_{n} \rho^{n}
$$

$$
\phi_{1}(\rho)=\sum_{n=0}^{\infty} a_{n} \rho^{n}, \quad \phi_{2}(\rho)=\sum_{n=-1}^{-\infty} a_{n} \rho^{n} .
$$

The integral equation (1) is thus equivalent to the pair of equations

$$
\begin{gathered}
\int_{0}^{\infty} f_{1}(t) K(\rho, t) d t=\phi_{1}(\rho), \quad 0<\rho<a, \\
\int_{0}^{\infty} f_{2}(t) K(\rho, t) d t=\phi_{2}(\rho), \quad \rho>b,
\end{gathered}
$$

where $f(\rho)=f_{1}(\rho)+f_{2}(\rho), b<\rho<a$ and

$$
f_{1}+f_{2}=0, \quad 0<\rho<b, \quad \rho>a .
$$


It can be shown $(7,8)$ that

$$
\begin{aligned}
K(\rho, t)=\frac{4}{(\rho t)^{n}} \int_{0}^{\min (\rho, t)} \frac{w^{2 n} d w}{\left(\rho^{2}-w^{2}\right)^{\frac{1}{2}}\left(t^{2}-w^{2}\right)^{\frac{1}{2}}}=4(\rho t)^{n} \\
\qquad \int_{\max (\rho, t)}^{\infty} \frac{w^{-2 n} d w}{\left(w^{2}-\rho^{2}\right)^{\frac{1}{2}}\left(w^{2}-t^{2}\right)^{\frac{1}{2}}}
\end{aligned}
$$

and it follows from equation (5) that equations (2) and (3) become

$$
\begin{gathered}
\int_{0}^{\rho} \frac{w^{2 n}}{\left(\rho^{2}-w^{2}\right)^{\frac{1}{2}}} \int_{w}^{\infty} \frac{t^{-n} f_{1}(t) d t d w}{\left(t^{2}-w^{2}\right)^{\frac{1}{2}}}=\frac{1}{4} \rho^{n} \phi_{1}(\rho), \quad 0<\rho<a, \\
\int_{\rho}^{\infty} \frac{w^{-2 n}}{\left(w^{2}-\rho^{2}\right)^{\frac{1}{2}}} \int_{0}^{w} \frac{t^{n} f_{2}(t) d t d w}{\left(w^{2}-t^{2}\right)^{\frac{1}{2}}}=\frac{1}{4} \rho^{-n} \phi_{2}(\rho), \quad \rho>b \ldots .
\end{gathered}
$$

Inversion of equations (6) and (7) gives

$$
\begin{gathered}
\int_{\rho}^{\infty} \frac{t^{-n} f_{1}(t) d t}{\left(t^{2}-\rho^{2}\right)^{\frac{1}{2}}}=\frac{\rho^{-2 n}}{2 \pi} \frac{d}{d \rho} \int_{0}^{\rho} \frac{t^{n+1} \phi_{1}(t) d t}{\left(\rho^{2}-t^{2}\right)^{\frac{1}{2}}}=g_{1}(\rho), \quad 0<\rho<a, \\
\int_{0}^{\rho} \frac{t^{n} f_{2}(t) d t}{\left(\rho^{2}-t^{2}\right)^{\frac{1}{2}}}=-\frac{\rho^{2 n}}{2 \pi} \frac{d}{d \rho} \int_{\rho}^{\infty} \frac{t^{1-n} \phi_{2}(t) d t}{\left(t^{2}-\rho^{2}\right)^{\frac{1}{2}}}=g_{2}(\rho), \quad p>b .
\end{gathered}
$$

Two new functions $h_{1}(\rho), h_{2}(\rho)$ are now defined by the equations

$$
\begin{aligned}
& h_{1}(\rho)=\int_{\rho}^{\infty} \frac{t^{-n} f_{2}(t) d t}{\left(t^{2}-\rho^{2}\right)^{\frac{1}{2}}}, \quad \rho>a, \quad . \\
& h_{2}(\rho)=\int_{0}^{\rho} \frac{t^{n} f_{1}(t) d t}{\left(\rho^{2}-t^{2}\right)^{\frac{1}{2}}}, \quad 0<\rho<b .
\end{aligned}
$$

It now follows from equations (4) and (10) that

$$
\int_{\rho}^{\infty} \frac{f_{1}(t) t^{-n} d t}{\left(t^{2}-\rho^{2}\right)^{\frac{1}{2}}}=-h_{1}(\rho), \quad \rho>a .
$$

Inversion of equations (8) and (12) gives

$$
f_{1}(\rho)=-\frac{2}{\pi} \rho^{n} \frac{d}{d \rho}\left[-\int_{a}^{\infty} \frac{t h_{1}(t) d t}{\left(t^{2}-\rho^{2}\right)^{\frac{1}{2}}}+\int_{\rho}^{a} \frac{t g_{1}(t) d t}{\left(t^{2}-\rho^{2}\right)^{\frac{1}{2}}}\right], \quad 0<\rho<a,
$$

and from equations (11) and (13) it now follows that

$h_{2}(\rho)=-\frac{2}{\pi} \int_{0}^{\rho} \frac{t^{2 n}}{\left(\rho^{2}-t^{2}\right)^{\frac{1}{2}}}\left[-t \int_{a}^{\infty} \frac{w h_{1}(w) d w}{\left(w^{2}-t^{2}\right)^{\frac{3}{2}}}+\frac{d}{d t} \int_{t}^{a} \frac{w g_{1}(w) d w}{\left(w^{2}-t^{2}\right)^{\frac{1}{2}}}\right] d t$,

We have that

$$
0<\rho<b \text {. }
$$

$$
\begin{aligned}
& \int_{0}^{\rho} \frac{t^{2 n}}{\left(\rho^{2}-t^{2}\right)^{\frac{1}{2}}} \frac{d}{d t} \int_{t}^{a} \frac{w g_{1}(w) d w d t}{\left(w^{2}-t^{2}\right)^{\frac{1}{2}}} \\
& \quad=\int_{0}^{\rho} \frac{t^{2 n+1}}{\left(\rho^{2}-t^{2}\right)^{\frac{1}{2}}}\left[\frac{-g_{1}(a)}{\left(a^{2}-t^{2}\right)^{\frac{1}{2}}}+\int_{t}^{a} \frac{g_{1}^{\prime}(w) d w}{\left(w^{2}-t^{2}\right)^{\frac{1}{2}}}\right] d t,
\end{aligned}
$$


and

$$
\begin{aligned}
\int_{0}^{\rho} \frac{t^{2 n+1}}{\left(\rho^{2}-t^{2}\right)^{\frac{1}{2}}} \int_{t}^{a} \frac{g_{1}^{\prime}(w) d w d t}{\left(w^{2}-t^{2}\right)^{\frac{1}{2}}}=\int_{0}^{\rho}\left[\int_{t}^{\rho}+\int_{\rho}^{a}\right] \frac{g_{1}^{\prime}(w) t^{2 n+1} d w d t}{\left(\rho^{2}-t^{2}\right)^{\frac{1}{2}}\left(w^{2}-t^{2}\right)^{\frac{1}{2}}} \\
=\left[\int_{0}^{\rho} \int_{0}^{w}+\int_{\rho}^{a} \int_{0}^{\rho}\right] \frac{t^{2 n+1} g_{1}^{\prime}(w) d t d w}{\left(\rho^{2}-t^{2}\right)^{\frac{1}{2}}\left(w^{2}-t^{2}\right)^{\frac{1}{2}}} \\
=\int_{0}^{a} g_{1}^{\prime}(w)\left[\int_{0}^{\min (\rho, w)} \frac{t^{2 n+1} d t}{\left(\rho^{2}-t^{2}\right)^{\frac{1}{2}}\left(w^{2}-t^{2}\right)^{\frac{1}{2}}}\right] d w .
\end{aligned}
$$

From equations (A1), (A2), (15) and (16) it follows that equation (14) may be re-written as

$$
\begin{aligned}
h_{2}(\rho) & =\frac{n !}{\sqrt{\pi \Gamma\left(n+\frac{3}{2}\right)}} \rho^{2 n+1} \int_{a}^{\infty} \frac{h_{1}(w)}{\left(w^{2}-\rho^{2}\right)} F\left(\frac{1}{2}, n, n+\frac{3}{2}, \frac{\rho^{2}}{w^{2}}\right) d w \\
& +\frac{g_{1}(a)}{\sqrt{ } \pi} \frac{(\rho a)^{2 n+1}}{\left(\rho^{2}+a^{2}\right)^{2 n+1}} \frac{n !}{\Gamma\left(n+\frac{3}{2}\right)} F\left(\frac{n+1}{2}, \frac{n}{2}+1, n+\frac{3}{2}, \frac{4 \rho^{2} a^{2}}{\left(\rho^{2}+a^{2}\right)^{2}}\right) \\
& -\frac{n !}{\sqrt{\pi \Gamma\left(n+\frac{3}{2}\right)}} \int_{0}^{a} \frac{g_{1}^{\prime}(w)(\rho w)^{2 n+1}}{\left(\rho^{2}+w^{2}\right)^{n+1}} \\
& F\left(\frac{n+1}{2}, \frac{n}{2}+1, n+\frac{3}{2}, \frac{4 \rho^{2} w^{2}}{\left(\rho^{2}+w^{2}\right)^{2}}\right) d w \quad 0<\rho<b .
\end{aligned}
$$

Equation (17) may be simplified to give

$$
\begin{aligned}
h_{2}(\rho)= & \frac{n ! \rho^{2 n+1}}{\sqrt{ } \pi \Gamma\left(n+\frac{3}{2}\right)}\left[\int_{a}^{\infty} \frac{h_{1}(w)}{\left(w^{2}-\rho^{2}\right)} F\left(\frac{1}{2}, n, n+\frac{3}{2}, \frac{\rho^{2}}{w^{2}}\right) d w\right. \\
& \left.-\frac{d}{d \rho} \rho \int_{0}^{a} \frac{w^{2 n} g_{1}(w)}{\left(\rho^{2}+w^{2}\right)^{n+1}} F\left(\frac{n+1}{2}, \frac{n}{2}+1, n+\frac{3}{2}, \frac{4 \rho^{2} w^{2}}{\left(\rho^{2}+w^{2}\right)^{2}}\right) d w\right], \\
& 0<\rho<b .
\end{aligned}
$$

It follows from equations (4) and (11) that

$$
\int_{0}^{\rho} \frac{t^{n} f_{2}(t) d t}{\left(\rho^{2}-t^{2}\right)^{\frac{1}{2}}}=-h_{2}(\rho), \quad 0<\rho<b .
$$

and hence, from equations (9), (10), (19),

$$
h_{1}(\rho)=\frac{2}{\pi} \int_{\rho}^{\infty} \frac{t^{-2 n}}{\left(t^{2}-\rho^{2}\right)^{\frac{1}{2}}}\left[t \int_{0}^{b} \frac{w h_{2}(w) d w}{\left(t^{2}-w^{2}\right)^{\frac{3}{2}}}+\frac{d}{d t} \int_{b}^{t} \frac{w g_{2}(w) d w}{\left(t^{2}-w^{2}\right)^{\frac{1}{2}}}\right] d t \rho>a, \ldots
$$

It follows after slight manipulation that

$$
\int_{\rho}^{\infty} \frac{t^{-2 n}}{\left(t^{2}-\rho^{2}\right)^{\frac{1}{2}}} \frac{d}{d t} \int_{b}^{t} \frac{w g_{2}(w) d w d t}{\left(t^{2}-w^{2}\right)^{\frac{1}{2}}}=\frac{d}{d \rho}\left\{\rho \int_{\rho}^{\infty} \frac{t^{-2 n-1}}{\left(t^{2}-\rho^{2}\right)^{\frac{1}{2}}} \int_{b}^{t} \frac{w g_{2}(w) d w d t}{\left(t^{2}-w^{2}\right)^{\frac{1}{2}}}\right\}
$$


and by an analysis similar to that of equation (16) it can be shown that

$\int_{\rho}^{\infty} \int_{b}^{t} \frac{t^{-2 n-1} w g_{2}(w) d w d t}{\left(t^{2}-\rho^{2}\right)^{\frac{1}{2}}\left(t^{2}-w^{2}\right)^{\frac{1}{2}}}=\int_{b}^{\infty} w g_{2}(w)\left[\int_{\max (\rho, w)}^{\infty} \frac{t^{-2 n-1} d t}{\left(t^{2}-\rho^{2}\right)^{\frac{1}{2}}\left(t^{2}-w^{2}\right)^{\frac{1}{2}}}\right] d w$.

From equations (A3), (A4), (21) and (22) it is seen that equation (20) can be re-written

$$
\begin{aligned}
h_{1}(\rho)= & \frac{n !}{\sqrt{\pi \Gamma\left(n+\frac{3}{2}\right)}}\left[\rho^{-2 n} \int_{0}^{b} \frac{w h_{2}(w)}{\left(\rho^{2}-w^{2}\right)} F\left(\frac{1}{2}, n, n+\frac{3}{2}, \frac{w^{2}}{\rho^{2}}\right) d w\right. \\
& \left.+\frac{d}{d \rho}\left\{\rho \int_{b}^{\infty} \frac{w g_{2}(w)}{\left(\rho^{2}+w^{2}\right)^{n+1}} F\left(\frac{n+1}{2}, \frac{n}{2}+1, n+\frac{3}{2}, \frac{4 \rho^{2} w^{2}}{\left(\rho^{2}+w^{2}\right)^{2}}\right) d w\right\}\right],
\end{aligned}
$$

$\rho>a$.

For $n=0$ equations (18) and (23) may be identified with those obtained by Gubenko and Mossakovskii (3) for the electrostatic problem.

\section{A more general class of boundary value problem}

We now consider the more general type of integral equation

$$
\int_{b}^{a} f(t)\left[K(\rho, t)+K_{1}(\rho, t)\right] d t=\phi(\rho), \quad b<\rho<a .
$$

Integral equations of the general form of equation (24) occur in boundary value problems which can in some sense be regarded as perturbations on the electrostatic problem for the annulus. Examples of such problems are the diffraction of an acoustic wave by a soft annulus and the electrostatic problem for an annulus in a circular cylinder.

In the notation of Section 2 equation (24) is equivalent to

$$
\begin{array}{ll}
\int_{0}^{\infty} f_{1}(t) K(\rho, t) d t=\phi_{1}(\rho)-\int_{0}^{\infty} f_{1}(t) K_{1}(\rho, t) d t, & 0<\rho<a, \\
\int_{0}^{\infty} f_{2}(t) K(\rho, t) d t=\phi_{2}(\rho)-\int_{0}^{\infty} f_{2}(t) K_{1}(\rho, t) d t, & \rho>b .
\end{array}
$$

It now follows immediately from equation (5) and the analysis of (6) that equations (25) and (26) can be reduced to

$$
\begin{array}{ll}
\rho^{2 n} S_{1}(\rho)=g_{1}(\rho)-\frac{1}{4} \int_{0}^{\infty} S_{1}(t) M_{1}(\rho, t) d t, & 0<\rho<a, \\
\rho^{-2 n} S_{2}(\rho)=g_{2}(\rho)-\frac{1}{4} \int_{0}^{\infty} S_{2}(t) M_{2}(\rho, t) d t, & \rho>b, \ldots
\end{array}
$$

where $S_{1}$ and $S_{2}$ are defined by the extreme left hand sides of equation (8) and (9), respectively. The functions $M_{1}$ and $M_{2}$ are defined by the relationships

$$
K_{1}(\rho, t)=\frac{1}{(\rho t)^{n}} \int_{0}^{\rho} \int_{0}^{t} \frac{M_{1}(u, v) d u d v}{\left(\rho^{2}-u^{2}\right)^{ \pm}\left(t^{2}-v^{2}\right)^{\frac{1}{2}}}
$$




$$
K_{2}(\rho, t)=(\rho t)^{n} \int_{\rho}^{\infty} \int_{t}^{\infty} \frac{M_{2}(u, v) d u d v}{\left(u^{2}-\rho^{2}\right)^{\frac{1}{2}}\left(v^{2}-t^{2}\right)^{\frac{1}{2}}}
$$

It follows from equations (4), (10), (11) that equations (27) and (28) are equivalent to

$$
\begin{aligned}
& \rho^{2 n} S_{1}(\rho)=g_{1}(\rho)-\frac{1}{4} \int_{0}^{a} S_{1}(t) M_{1}(\rho, t) d t+\frac{1}{4} \int_{a}^{\infty} h_{1}(t) M_{1}(\rho, t) d t, \quad 0<\rho<a, \\
& \rho^{-2 n} S_{2}(\rho)=g_{2}(\rho)+\frac{1}{4} \int_{0}^{b} h_{2}(t) M_{2}(\rho, t) d t-\frac{1}{4} \int_{b}^{\infty} S_{2}(t) M_{2}(\rho, t) d t, \quad \rho>b .
\end{aligned}
$$

The analysis of Section 2 shows that $h_{1}$ and $h_{2}$ will still satisfy equations (23) and (18) provided that $g_{1}$ and $g_{2}$ in these equations are now replaced by $S_{1}$ and $S_{2}$ respectively. These two equations together with equations (31) and (32) thus give a set of four Fredholm integral equations for the functions $h_{1}, h_{2}$, $S_{1}, S_{2}$.

In most boundary value problems where equation (24) occurs the function $K_{1}(\rho, t)$ will generally involve only terms of the form $J_{n}(\lambda \rho) J_{n}(\lambda t)$ and it follows from Sonine's finite and infinite integral formulae that, for $K_{1}$ of this form,

$$
\begin{aligned}
& M_{1}(u, v)=\frac{2 \lambda}{\pi}(u v)^{n+\frac{1}{2}} J_{n-\frac{1}{2}}(\lambda u) J_{n-\frac{1}{2}}(\lambda v), \\
& M_{2}(u, v)=\frac{2 \lambda}{\pi}(u v)^{\frac{1}{2}-n} J_{n+\frac{1}{2}}(\lambda u) J_{n+\frac{1}{2}}(\lambda v) .
\end{aligned}
$$

\section{Appendix}

Expressions for various definite integrals are required in the analysis of Section $\mathbf{2}$ and for convenience the relevant formulæ have been collected together in this Appendix.

The required formulæ are

$$
\begin{aligned}
& \int_{0}^{\rho} \frac{t^{2 n+1} d t}{\left(\rho^{2}-t^{2}\right)^{\frac{1}{2}\left(w^{2}-t^{2}\right)^{\frac{3}{2}}}} \quad=\frac{n ! \sqrt{ } \pi \rho^{2 n+1}}{2 \Gamma\left(n+\frac{3}{2}\right) w\left(w^{2}-\rho^{2}\right)} F\left(\frac{1}{2}, n ; n+\frac{3}{2} ; \frac{\rho^{2}}{w^{2}}\right), w>\rho, \ldots \ldots \ldots \\
& \int_{0}^{\min (\rho, w)} \frac{t^{2 n+1} d t}{\left(\rho^{2}-t^{2}\right)^{\frac{1}{2}}\left(w^{2}-t^{2}\right)^{\frac{1}{2}}} \\
& \quad=\frac{1}{2} \frac{n ! \sqrt{ } \pi(\rho w)^{2 n+1}}{\left(\rho^{2}+w^{2}\right)^{n+1}} F\left(\frac{n+1}{2}, \frac{n}{2}+1 ; n+\frac{3}{2} ; \frac{4 \rho^{2} w^{2}}{\left(\rho^{2}+w^{2}\right)^{2}}\right) \ldots \ldots(A) \\
& \int_{\rho}^{\infty} \frac{t^{1-2 n} d t}{\left(t^{2}-\rho^{2}\right)^{\frac{1}{2}}\left(t^{2}-w^{2}\right)^{\frac{3}{2}}} \quad=\frac{n ! \sqrt{ } \pi \rho^{-2 n}}{2 \Gamma\left(n+\frac{3}{2}\right)\left(\rho^{2}-w^{2}\right)} F\left(\frac{1}{2}, n, n+\frac{3}{2}, \frac{w^{2}}{\rho^{2}}\right), \quad w<\rho, \ldots \ldots \ldots . . .
\end{aligned}
$$




$$
\begin{aligned}
& \int_{\max (\rho, w)}^{\infty} \frac{t^{-2 n-1} d t}{\left(t^{2}-\rho^{2}\right)^{\frac{1}{2}}\left(t^{2}-w^{2}\right)^{\frac{1}{2}}} \\
& \quad=\frac{n ! \sqrt{ } \pi}{2 \Gamma\left(n+\frac{3}{2}\right)\left(\rho^{2}+w^{2}\right)^{n+1}} F\left(\frac{n+1}{2}, \frac{n}{2}+1, n+\frac{3}{2}, \frac{4 \rho^{2} w^{2}}{\left(\rho^{2}+w^{2}\right)^{2}}\right) .(A
\end{aligned}
$$

Equation (A1) follows immediately from Euler's integral for the hypergeometric function and (A2) is a direct consequence of Euler's integral and Kummer's quadratic transformation formula. A derivation has been given by the author (9). Equations (A3), (A4) follow from equations (A1), (A2) by an obvious change of variable.

For small values of the integer $n$ the hypergeometric functions on the right hand sides of the equations (A1, A4) can be expressed as fairly simple forms involving elementary functions.

\section{REFERENCES}

(1) W. E. Williams, Q. J. Mech. App. Math., 16 (1963), 205.

(2) J. C. CoOKe, Q. J. Mech. App. Math., 16 (1963), 193.

(3) V. S. Gubenko and V. I. MossakovskiI, P.M.M. 24 (1960), 334.

(4) W. D. Collins, Archive Rat. Mech. Anal. 11 (1962), 122.

(5) W. E. Williams, to appear, Proc. Camb. Phil. Soc.

(6) W. E. Williams, ZAMP 13 (1962), 133.

(7) E. T. Copson, Proc. Edin. Math. Soc. 8 (1947), 14.

(8) H. C. Levey and P. E. Wynters, Proc. Roy. Soc. A 268 (1962), 390.

(9) W. E. Williams, Proc. Camb. Phil. Soc., 59 (1963), 589

\section{Department OF APPLiEd Mathematics}

THE UNIVERSITY

LIVERPOOL 